\title{
FREE GROUPS AND HANDLEBODIES
}

\section{J. J. ANDREWS ${ }^{1}$ AND M. L. CURTIS ${ }^{1}$}

In this note we state a conjecture about free groups and give some topological consequences which would follow if the conjecture is true. The conjecture seems to be a natural extension of a theorem of Nielsen, and the main topological consequence is the theorem that regular neighborhoods of contractible 2-dimensional subcomplexes of combinatorial 5-manifolds are 5-cells. This has some interesting consequences relative to the 3 - and 4-dimensional Poincaré conjectures.

The referee has remarked on a weakening of the conjecture and on the relation of the topological problem to a more general problem as follows:

"The results of the paper follow from a somewhat weaker conjecture: Suppose $P$ is $\left(x_{1}, \cdots, x_{n}: r_{1}, \cdots, r_{n}\right)$, and is a presentation of the trivial group; define an operation of type (i), (ii), (iii), (iv) on this presentation to be the result of applying such an operation as defined in the paper on the set of relators. An operation of type (v) will consist of adding an additional generator, say $y$, and the additional relator $y$; an operation of type (vi) will be the inverse of an operation of type (v). The weaker conjecture would be that $P$ can be reduced to the empty presentation by a finite sequence of operations of types (i)-(vi).

"The problem is related to the question: If the 2-dimensional polyhedra $P$ and $Q$ have the same simple homotopy type, can they both be embedded in a 3-dimensional polyhedron $Z$ such that $Z$ geometrically contracts to $P$ and $Z$ geometrically contracts to $Q$ ? If you replace " 2 " and " 3 " by " $n$ " and " $n+1$," this question has the affirmative answer for $n$ greater than 2 , according to J. H. C. Whitehead in Simplicial spaces, nuclei, and m-groups. It is equivalent to the above conjecture when $P$ is a contractible 2-dimensional polyhedron and $Q$ is a point."

1. A conjecture about free groups. Let $F$ be the free group on $x_{1}, \cdots, x_{n}$. If $a_{1}, \cdots, a_{p}$ is any (finite) set of elements of $F$, then a Nielsen operation on $a_{1}, \cdots, a_{p}$ changes $a_{1}, \cdots, a_{p}$ to $\bar{a}_{1}, \cdots, \bar{a}_{p}$ in one of the following three ways:

(i) $\bar{a}_{1}=a_{1}^{-1}, \bar{a}_{2}=a_{2}, \cdots, \bar{a}_{p}=a_{p}$.

(ii) Interchange $a_{1}$ and $a_{i}$, leaving others the same.

(iii) $\bar{a}_{1}=a_{1} a_{2}$, all others fixed.

Received by the editors September 20, 1963.

1 Partially supported by NSF, GP 626. 
TheOREM 1 (NIELSEN). If $F$ is free on $x_{1}, \cdots, x_{n}$ and also free on $y_{1}, \cdots, y_{n}$, then a finite sequence of Nielsen operations will change $x_{1}, \cdots, x_{n}$ to $y_{1}, \cdots, y_{n}$. (That is, Nielsen operations generate the group of automorphisms of $F$.)

The normal closure of a set $A$ in a group $G$ is the smallest normal (in $G$ ) subgroup containing $A$. If $F$ is free on $x_{1}, \cdots, x_{n}$, and the set $r_{1}, \cdots, r_{n}$ is such that its normal closure is $F$, then it may not be possible to change $r_{1}, \cdots, r_{n}$ to $x_{1}, \cdots, x_{n}$ by Nielsen operations. However, we suspect that this is possible if a fourth operation is allowed.

(iv) $\bar{a}_{1}=g a_{1} g^{-1}$, all others fixed, where $g$ is any element of $F$.

CONJECTURE. If $F$ is free on $x_{1}, \cdots, x_{n}$ and the normal closure of $r_{1}, \cdots, r_{n}$ is $F$, then $r_{1}, \cdots, r_{n}$ may be changed to $x_{1}, \cdots, x_{n}$ by a finite sequence of operations of types (i), (ii), (iii), (iv).

Some results showing that the conjecture is true in some special cases will be published later.

\section{Topological consequences.}

THEOREM 2. Let $K$ be a finite contractible 2-dimensional subcomplex of a combinatorial 5-manifold $M$, and let $N$ be any regular neighborhood of $K$ in $M$. If the conjecture is true then $N$ is a 5-cell.

According to Whitehead [5], any two regular neighborhoods of $K$ in $M$ are combinatorially equivalent, so it suffices to look at a specific regular neighborhood. We will do this by building up $N$ as a handlebody.

Let $T$ be a maximal tree in $K$. Then both $M$ and $N$ are unchanged by collapsing $T$ to a point, and $K$ becomes a bouquet of loops with disks attached by their boundaries. (Since $K$ is contractible, there will be the same number of disks as there are loops.) Let $L$ be the part of $N$ consisting of the regular neighborhood of the bouquet of loops. This is just the 5-cell regular neighborhood of the vertex with a 1-handle added for each loop.

The disks of $K$ will intersect the boundary of $L$ in disjoint simple closed curves. The neighborhood $N$ can be considered to be made up of $L$ together with the disks of $K$ thickened into 5-cells. These thickened disks are 2-handles which are regular neighborhoods in $M$ of the parts of the disks of $K$ which lie outside of $L$.

For the proof of the theorem, we need a couple of lemmas.

Lemma 1. If two simple closed curves in a 4-manifold $Q$ are homotopic, then they are ambient isotopic. (All pw. l.) 
This is a consequence of a result of Guggenheim [1]. It is also easily proved by imbedding the mapping cylinder of the homotopy by the Penrose-Whitehead-Zeeman piping technique [4], and then using results of Whitehead.

From this lemma we easily get the next result.

Lemma 2. The ambient isotopy class of a simple closed curve on the boundary of $L$ is determined by the word it represents in the free group generated by the loops of $K$.

This follows from Lemma 1 because the homotopy class of the curve is determined by the word which it represents.

The lemma is false in one lower dimension, as may be seen by an example due to Mazur [2]. Here there is one loop, and a curve $x x x^{-1}$ which is not ambient isotopic to the curve $x$, whereas in the free group generated by $x$, these are the same. Essentially Lemma 2 means that in working with the curves on the boundary of $L$, any indicated cancellations can be performed.

Let $r_{1}, \cdots, r_{n}$ be the curves on the boundary of $L$ (the intersections of the disks of $K$ with the boundary of $L$ ). Let $F$ be the group free on loops $x_{1}, \cdots, x_{n}$, so that according to Lemma 2 , each $r_{i}$ is represented as a word in the $r$ 's, i.e., as an element of $F$. Any regular neighborhood obtained from $L$ by adding these disks and then thickening them will be said to realize $r_{1}, \cdots, r_{n}$.

If $N$ realizes $r_{1}, \cdots, r_{n}$ and $s_{1}, \cdots, s_{n}$ is obtained from $r_{1}, \cdots, r_{n}$ by a finite sequence of operations of the four types, then there is a manifold homeomorphic to $N$ which realizes $s_{1}, \cdots, s_{n}$.

Smale has shown this to be true for the three Nielsen operations, so it suffices to check it for the fourth operation, namely conjugation. But $g h g^{-1}$ is homotopic, and hence ambient isotopic to $h$ in the boundary of $L$. This determines an isotopy of $N$ onto the new manifold. (Indeed, all of the operations except inverses may be done by isotopies.)

Now if the conjecture is true, then $r_{1}, \cdots, r_{n}$ can be changed to $x_{1}, \cdots, x_{n}$ by the four operations. Hence $N$ is homeomorphic to a manifold realizing $x_{1}, \cdots, x_{n}$. But a manifold realizing $x_{1}, \cdots, x_{n}$ turns out to be a regular neighborhood of a 2-complex which has $n$ loops, each filled with exactly one disk. Such a complex is collapsible, so that by Whitehead's theorem, the manifold is a cell. This completes the proof of Theorem 2 .

COROLlary 1. If a homotopy 4-sphere has a 2-spine (and the conjecture is true), then it is a 4-sphere. 
To see this, let $S$ be a combinatorial manifold which is a homotopy 4-sphere. Our hypothesis that it have a 2 -spine means that if a small 4 -ball is removed, the resulting manifold $M$ collapses to a 2-dimensional subcomplex $K$. Clearly $K$ is contractible.

Consider $M \times I$. This is a 5 -manifold which is a regular neighborhood of $K$. By Theorem 2, $M \times I=I^{5}$, so the boundary of $M \times I$ is $S^{4}$, and this is just two copies of $M$ attached by their boundary 3-spheres. This common boundary 3 -sphere is collared, so that $M=I^{4}$. It follows that $S=S^{4}$.

Corollary 2. If the 3-dimensional Poincaré conjecture is false (and the conjecture made here is true), then a counterexample exists in 4-space. In particular, if $F^{3}$ is a fake 3-cell, then $2 F^{3}$ is a fake 3-sphere which may be imbedded in a 4-space.

Suppose $S$ is a homotopy 3-sphere which is not homeomorphic with $S^{3}$. Then if $F^{3}$ is obtained from $S$ by removing a small 3-cell, $F^{3}$ is not a 3 -cell, but it does have a contractible 2 -spine $K$.

Consider $F^{3} \times I^{2}$. This 5 -manifold has $K$ as 2 -spine, so that it is a 5 -cell. Its boundary 4 -sphere is easily seen to contain $2 F^{3}$.

\section{REFERENCES}

1. V. K. A. M. Guggenheim, Piecewise linear isotopies and embedding of elements and spheres. I, II, Proc. London Math. Soc. (3) 3 (1953), 129-152.

2. B. Mazur, A note on some contractible 4-manifolds, Ann. of Math. (2) 73 (1961), 221-228.

3. R. Penrose, J. H. C. Whitehead and E. C. Zeeman, Imbedding of manifolds in euclidean space, Ann. of Math. (2) 73 (1961), 613-623.

4. J. H. C. Whitehead, Simplicial spaces, nuclei and m-groups, Proc. London Math. Soc. (2) 45 (1939), 243-327.

Florida State University 